\title{
Peering into the heart of a black hole
}

Quantum mechanics might be capable of stripping bare a black hole to reveal the mysterious and unseeable 'singularity' that exists at its heart ${ }^{1}$, say George Matsas and André da Silva of the São Paulo State University in Brazil.

It has long been suspected that these singularities - where the known laws of physics break down - are always decorously veiled behind the 'event horizon', a boundary beyond which light cannot escape from the fearsome gravitational pull of a black hole. Theoretically, nothing within an event horizon can ever be perceived or investigated by an outside observer, because no light can escape. So the singularities remain insulated from the rest of the Universe.

This amounts to what in 1969 physicist Roger Penrose called 'cosmic censorship', whereby the laws of physics conspire to save us from having to gaze on the unthinkable. According to Einstein's general theory of relativity, in the middle of a black hole, its mass collapses in on itself to form an infinitely small, infinitely dense point, where space-time itself is punctured. Even causality - the relation of a cause and its effect - breaks down, which seems to defy not only physics but logic. "Penrose's motivation seemed to be to preserve the decorum of physics," Matsas says.

But physicists have wondered whether event horizons are ever stripped away, leaving these absurdist singularities naked. One possibility, for example, is that the event horizon might vanish if a black hole spins very fast. Light and matter might then be flung out by centrifugal force.

In September, physicists Arlie Petters of Duke University in Durham, North Carolina, and Marcus Werner of the University of Cambridge, UK, proposed that singularities stripped naked by fast rotation should be detectable by astronomers because they act as very strong 'gravitational lenses', bending the light coming from stars behind them by their distortion of space-time. Petters

\section{"It is widely believed} that quantum gravity will unveil the structure of the singularities."

transfer enough angular momentum to the hole to overspin it into nakedness, a particle would have to approach the event horizon at such a high speed and such a glancing angle (similar to stroking the side of a spinning-top) that it wouldn't get sucked inside the event horizon in the first place.

No way of creating a naked singularity has yet been discovered within the framework of the classical physics described by the theory of relativity. But in a paper published last week, Matsas and da Silva propose that quantum mechanics, which normally applies only to very small objects, could subvert cosmic censorship. An electrically charged black hole spinning fast enough to be right on the brink of losing its event horizon might be pushed over the edge by gaining angular momentum in a non-classical way, they conclude. and Werner say that existing telescopes should have sufficient spatial resolution to spot naked singularities in the centre of our own Galaxy ${ }^{2}$.

But how could a black hole spin fast enough to bare its heart? It was shown in the 1970s that a black hole's spin cannot be increased by swallowing rotating objects, because the gain in angular momentum - the momentum caused by rotation - is generally balanced by the slowing influence of the extra mass. To



BURSTING DARK

ENERGY'S BUBBLE

Mysterious force could be an 'artefact' of a void in space.

www.nature.com/news

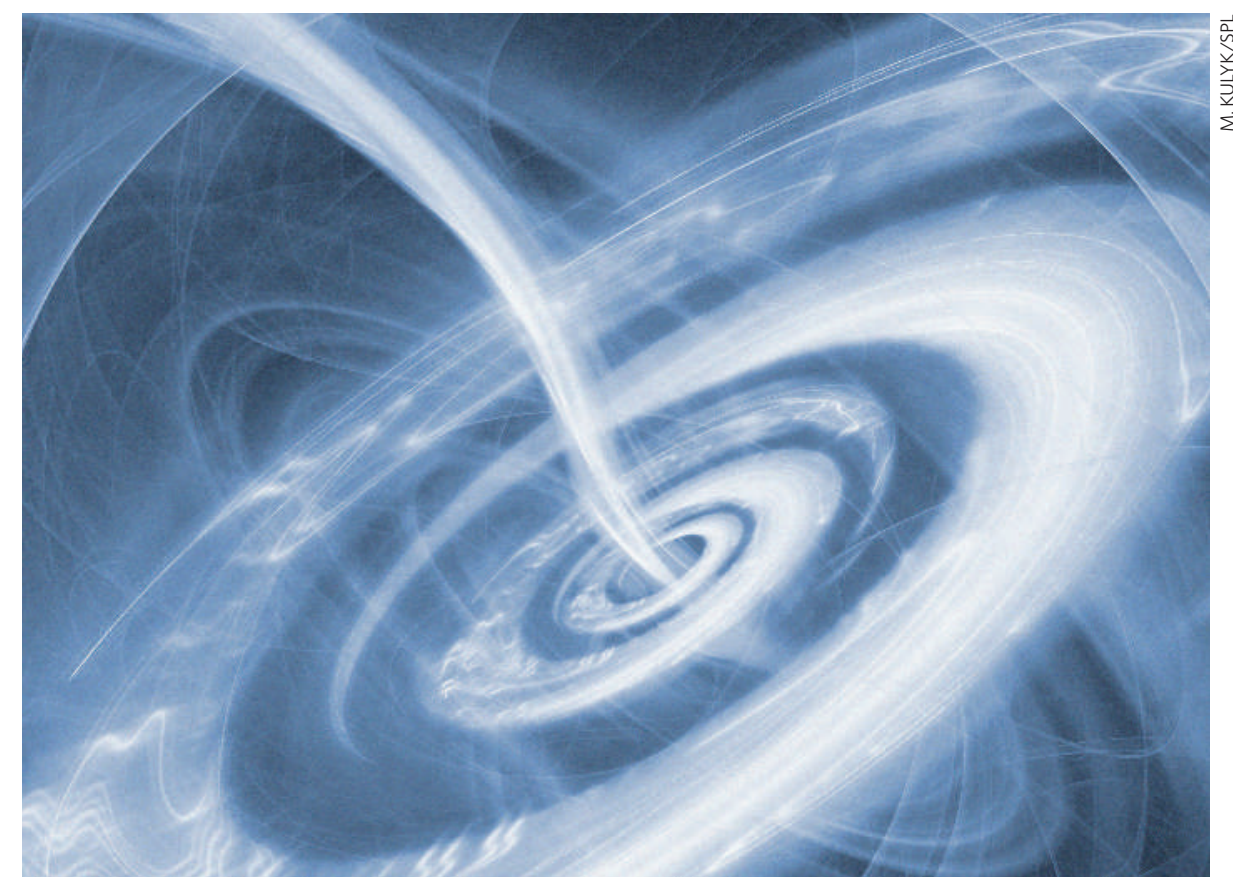

over the brink into nakedness. "It's a neat idea," says Petters. "One issue is whether such black holes exist, or if they will remain charged long enough for us to catch one, since charged black holes tend to become neutralized."

Matsas himself cautions that the work does not necessarily mean that cosmic censorship is violated in reality, because quantum theory is known to be incomplete. The general theory of relativity that explains gravity and predicts black holes, and the theory of quantum mechanics, are known to be fundamentally incompatible, and physicists hope that they might ultimately be reconciled in a quantum theory of gravity.

Whether such a theory would rescue cosmic censorship, says Matsas, remains to be seen. But he says that "we don't see any compelling reason to preclude the existence of naked singularities in the context of quantum gravity". Such an improved theory should in fact help make sense of what naked singularities are like in the first place. "It is widely believed that quantum gravity will unveil the structure of the singularities," says Matsas, adding that they will then probably seem "quite benign to physics" rather than the

\footnotetext{
1. Matsas, G. E. A. \& da Silva, A. R. R. Phys. Rev. Lett. 99, 181301 2. Werner, M. C. \& Petters, A. O. Phys. Rev. D 76, 064024
(2007). (2007).
} monstrosities they now seem to be.

Philip Ball

explained in the 1970s, Matsas and Silva's study finds that quantum particles could tunnel inside, and send the black hole of being able to 'tunnel' through barriers that, according to classical mechanics, they have forficient energy to pass over or through.

whereas classical particles with eno angular momentum to overspin a black hole can't get inside the event horizon for the rea- 